\title{
A Load Balancing Algorithm Using the Circulation of A Single Message Token
}

\author{
Jun Hwang, Woong J. Lee, Byong G. Lee, and Young S. Kim \\ School of Computer Science and Engineering, Seoul Women's University, \\ 126 Nowon-Gu GongReung-Dong Seoul, Korea, 139-774 \\ \{hjun,wjlee, byongl, yskim\}@swu.ac.kr
}

\begin{abstract}
We present an efficient load balancing algorithm which simplifies the system status information through the use of an information message (VISITOR) in distributed system. Using the proposed algorithm, information gathering for decision-making is possible with less number of messages than the one using existing load balancing algorithm. The proposed algorithm improves the performance of distributed systems over the existing algorithms, not only by exchanging fewer messages to gather the information for making decision on load balancing and migration, but also by automatically determining of node and when to migrate.
\end{abstract}

\section{Introduction}

Load Balancing Strategy (LBS) is an activity of process sharing performed by distributed processes. The activity is called as a global or distributed scheduling $[14,11]$ in that process allocation is targeted to all distributed processes while typical process scheduling such as FIFO, LRU, RR are called as a local scheduling. LBS minimizes response time and maximizes the throughput by considering a system as a whole virtual process. The performance of load balancing mainly depends on its algorithm, which can be categorized as following;

First, when a node has to inform its own load status to other nodes, or to select a node to migrate, load balancing algorithm takes necessary global information through message broadcasting and do process migration accordingly $[16,10]$. Second, a node utilizes the global information only for figuring out the load status of each node. For other decision-making, such as load balancing and migration, is done by heuristic information [16]. Third, each node does not need the global information from every node but from adjacent node only, and the load balancing is done by the judgment of a local node $[16,7,4]$.

These load balancing algorithms may cause the inundation of broadcasting messages in picking up of global information and in selecting a node for migration [1]. In this paper, proposed is an algorithm that performs 
the global information retrieval and load balancing through a single information message by simplifying the way of acquiring the global information and of making decision for migration.

Chapter 2 describes a load balancing algorithm, e.g., VISITOR algorithm, and reviews its concept and characteristics. Chapter 3 introduces VISITOR ( $¥-)$ which enhances the VISITOR algorithm. In chapter 4 , the simulation context and necessary parameters for VISITOR (II) are discussed, and the performance of the proposed algorithm is analyzed in comparison with VISITOR algorithm. Finally, Chapter 5 concludes the paper with a summary and future work.

\section{Load Balancing Algorithm}

\subsection{The Concept of VISITOR Algorithm}

Provided with the load information, each node compares its own load state with those of entire system. In case that load imbalance is detected by exceeding a neutral zone, process migration occurs from an overloaded node to an under-loaded node. According to which node requests the migration, different approaches can be used, e.g., 'Receiver-Initiative' or 'SenderInitiative'.

The VISITOR message contains the global information that represents the load status and migration information of each node. This message is circulated along the nodes at constant time interval and supplies necessary information to each node.

\subsection{VISITOR Algorithm}

To find out whether the load status of each node is overload or under-load, an average load value must be used. The average load value influences so much to the performance and efficiency of load balancing algorithm [6, 15]. The tracing function $\mathrm{H}$ for measurement of average load value is defined as follows;

$$
\begin{aligned}
\mathrm{H}^{*}\left(\mathrm{AVG}_{\mathrm{k}}\right) & =\mathrm{AVG}_{\mathrm{k}}+1 / \mathrm{m} *\left(\mathrm{LOCAL}_{\mathrm{AVG}}\right)+\mathrm{CD} ; f ; \quad(1) \\
& =\mathrm{AVG}_{\mathrm{k}+1} \\
\text { and } \mathrm{AVG}_{0} & =0
\end{aligned}
$$

where $\mathrm{AVG}_{\mathrm{k}}$ : average load value on VISITOR message after $\mathrm{k}^{\text {th }}$ circulation

$\mathrm{AVG}_{\mathrm{k}+1}$ : expected average load value after $\mathrm{k}+1$ circulation

$m \quad$ : number of nodes in the distributed system

LOCAL : measured value of local load (number of processes in local queue)

CD : communication latency time 
The function $\mathrm{H}$ is derived from the facts that the load value at a node influences to the global information at the ratio of $1 / \mathrm{m}$. Except from the information that is used in process migration, a control message is also necessary to run VISITOR algorithm as following.

- VISITOR: circulates along all the nodes in the connection during load balancing and provides the load balancing information.

- ACCEPT: permits migration of overloaded node to under-loaded node.

- RECOMM: does not participate in the migration, but informs the overloaded nodes that other nodes are under-loaded states.

Figure 1 depicts the structure of VISITOR message. VISITOR message fills with the information such as average load value, the least loaded node, and the most loaded node, and circulates along logical ring consecutively. As the VISITOR message passes through nodes, each node keeps its local load value and figures out load status of its own as to the whole system.

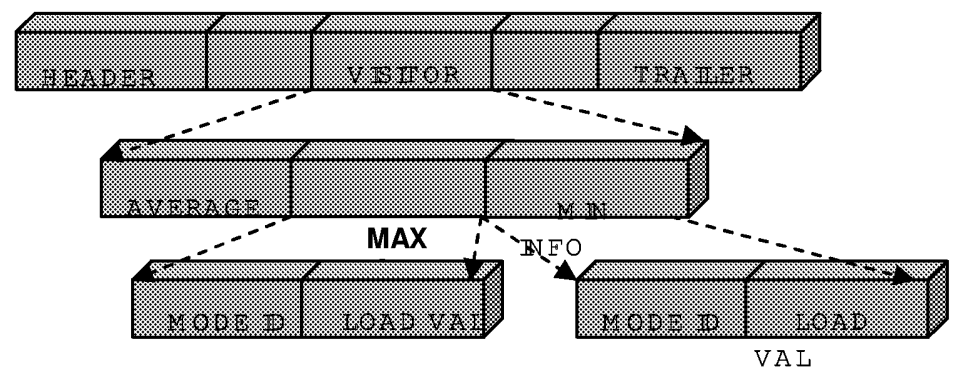

Fig. 1. The VISITOR message format

\section{VISITOR( $¥-)$ Algorithm}

Because VISITOR is complicated algorithm, it is a burden for a distributed system to take responsibility of load balancing. To lessen the burden, VISITOR ( $¥-)$ algorithm is suggested by modifying original VISITOR algorithm. The difference between VISITOR and VISITOR ( $¥-$ ) algorithm is that VISITOR $(¥-)$ adopts 'Receiver-Initiative' method (VISITOR uses 'Sender/Receiver-Initiative' method). It is known that if the communication latency is short and the node is not overloaded, 'Receiver-Initiative' method performs better than 'Sender/Receiver-Initiative' method. 


\subsection{Detailed Description of VISITOR ( $¥-)$ Algorithm}

In section 2.2, the heuristic function $\mathrm{H}$ was defined with equation (1). The function $\mathrm{H}$ is to calculate a new average load value with local load value and system-wise load value. However, with the function $\mathrm{H}$, average load value is amplified without any control as the number of nodes and communication latency increases, thus resulting in high variance of average value. Thus, a function $\mathrm{F}$ that is complimentary of function $\mathrm{H}$, is introduced.

$$
\begin{aligned}
\mathrm{F} & =\mathrm{AVG}_{\mathrm{K}}+\mathrm{ACE} * 1 / \mathrm{m} *\left(\mathrm{LOCAL}-\mathrm{AVG}_{\mathrm{K}}\right) \\
& =\mathrm{AVG}_{\mathrm{K}+1}
\end{aligned}
$$

and $\quad \mathrm{AVG}_{0}=0$,

$\mathrm{ACE}=\mathrm{C} /(\mathrm{LOCAL}-\mathrm{AVG}+\mathrm{C})$

where, LOCAL : number of process in local queue

$\mathrm{m}: \quad$ number of nodes in distributed system

ACE: Adaptive Control Element (ACE) to reduce the error in tracing new average

C : communication Delay Factor*Constant

The ACF module influences the result of average load value. The ACF prevents the load value from influencing other nodes' values without any controlling over the value. That is, if the local load value in $\mathrm{H}$ is extremely high or low, its load value influences average load-tracing value of other nodes at the ratio of $1 / \mathrm{m}$. This displacement occurs periodically and reacts sensitively if the state of entire distributed system changes. Therefore, a settlement is necessary in calculating the average load value of node. Adding a simple function called as ACF solves this problem. The influences of ACF on average load value should keep minimal, even as the difference increases [5]. In addition, VISITOR (II) includes message transmission information. The message format of VISITOR ( $¥-)$ is shown in figure 2 .

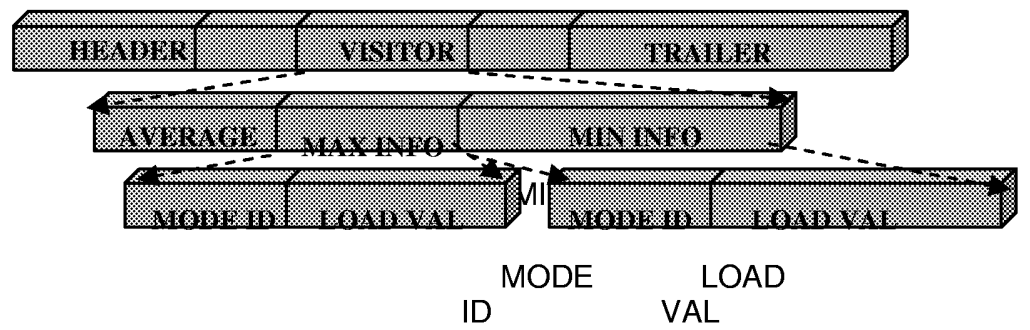

Fig. 2. VISITOR( $¥-)$ message format 


\subsection{VISITOR( $¥-)$ Algorithm}

VISITOR(II) algorithm for load balancing follows;

CASE 1: In case of a VISITOR( $¥-)$ message arriving,

- Calculate a new average load value through ACF and decide its own load status by averaging the load value.

- Execute the routine steps of load balancing.

CASE 2: In case of overload state,

- If there exist MIN information and its load value is less than average load value, choose a process from local queue.

- Migrate selected process to the under-loaded node.

- Eliminate the field information from MIN_INFO on VISITOR message.

- Pass the VISITOR ( $¥-)$ message to the next node.

CASE 3: In case of under-loaded state,

- If there exist MIN information and its load value is greater than average load value, insert its own node ID and load value information into MIN_INFO field.

- Send the VISITOR $(¥-)$ message to the next node.

CASE 4: In case of optimal loaded state,

- Send the VISITOR $(¥-)$ message to the next node.

CASE 5: In case of process arriving,

- Increase its own local load and put it into the local queue

\section{The Simulation and Analysis}

\subsection{The Environment of Simulation}

The simulation uses $5 *(\mathrm{M} / \mathrm{M} / 1)$ model with following assumptions.

(1) Fully-connected form of network topology is assumed.

(2)The node number of distributed system is 5 and VISITOR message circulates in a numerical order (clockwise or counterclockwise).

(3) Suppose that the load status of random node, average arrival rate and average service time is given from table 1 .

(4) Suppose the communication capacity of network system be $0.1 \mathrm{Mbps}$ basically.

(5) Physical or logical loss of message or any related error is ignored.

(6) The message transmission is done in the unit of packet, and the length of packet is 1,000 bits.

(7) Transmission delay that takes for transmitting one packet is $10 \mathrm{msec}$.

(8) Length of process (work) is $12 \mathrm{Kbits.}$

(9) Size of message is 4 Kbits after remote execution of process.

(10) Length of control message is 1 Kbits and duration time that stays for transmission and collection of information in a node is $10 \mathrm{msec}$.

(11) Length of VISITOR message is 1 Kbits and duration time for staying and collecting of information is $10 \mathrm{msec}$. 
Table 1. The load status of each node

\begin{tabular}{|c|r|r|r|r|r|}
\hline Load status & EU & UL & NL & OL & EO \\
\hline $\begin{array}{c}\text { Average } \\
\text { arrival time }\end{array}$ & 11.76 & 11.11 & 10.10 & 9.09 & 8.33 \\
\hline $\begin{array}{c}\text { Average } \\
\text { service time }\end{array}$ & 10.0 & 10.0 & 10.0 & 10.0 & 10.0 \\
\hline
\end{tabular}

EU (Extremely Under-loaded State), UL (Under-loaded State)

NL (Loaded Normally State), OL (Overloaded State)

EO (Extremely Overloaded State)

Communication Delay $=($ PACKET SIZE/0.1Mbps $) *($ PROCESS SIZE/1000)--- $(2)$

The equation (2) calculates communication delay time independently with the simulation environment [12]. The total communication time is achieved by dividing the size of process with the length of packet (1000bits), and by multiplying it with communication latency time, which takes for transmitting one packet. Although above equation appears to be simple and insufficient, it still fully represents the characteristics of the communication system. The communication latency time is artificially calculated by equation (2) after accepting a message from a random node. After intended latency time is elapsed, the message is sent to a target node. This model is simulated in a fully connected network environment. Otherwise, more refinement should be done to the model.

\subsection{The Performance of Average Load Value Tracing Function}

The real load value of the entire system is calculated by dividing the total number of processes in waiting queue at random time with the number of nodes. The trace value of VISITOR message is calculated by function F and $\mathrm{H}$ (Figure 3). The status of each node is assumed as shown in table 2.

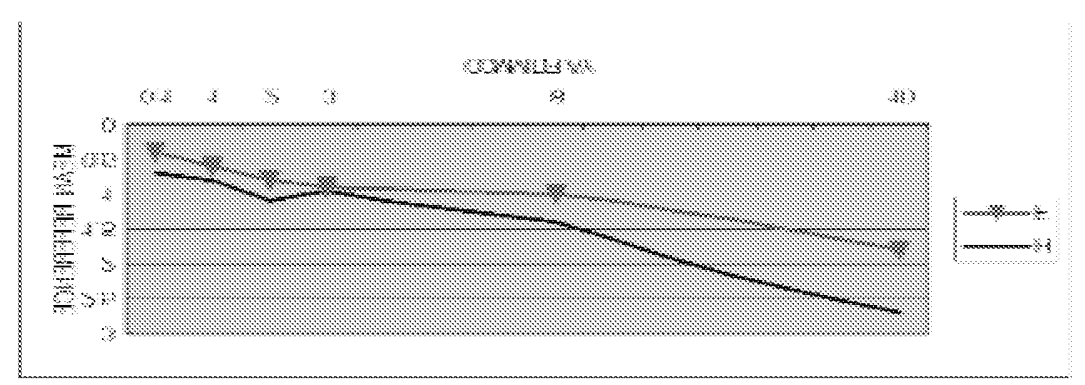

Fig. 3. The relation of real average load value (COMM.DELAY $=20 \mathrm{msec})$ and tracing average load value by function $\mathrm{F}$ and $\mathrm{H}$ respectively 
Table 2. The load status of each node

\begin{tabular}{|c|c|r|r|r|r|}
\hline Node number & 1 & 2 & 3 & 4 & 5 \\
\hline Load status & EU & OL & UL & EO & NL \\
\hline
\end{tabular}

To figure out the deviation between the real average load value and average tracing load value, the variation of standard deviation is used as depicted on figure 4. To get a accurate sample set, a sampling is done at a stable time of the system and the number of sample is about 3000 . To get average deviation value, the difference of real value and traced value is calculated [3].

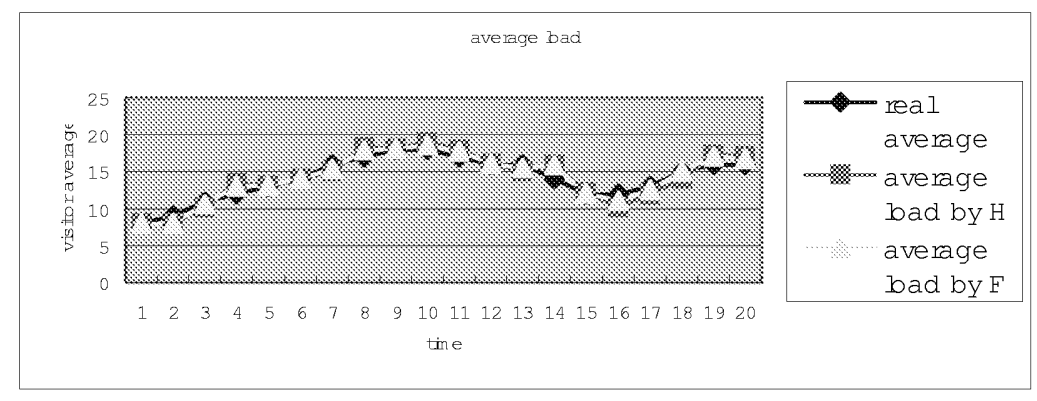

Fig. 4. The change of tracing value due to VISITOR passing delay

The actual average load value and sample mean of each trace function, $\mathrm{F}$ grows accordingly as communication latency increases. This means that the communication latency is proportional to error rate. Function F, compared to $\mathrm{H}$, is appeared to be low in speed of performance decrease due to the increase of communication costs. The performance degradation is stable with function $\mathrm{F}$ while function $\mathrm{H}$ showed sharp performance degradation. Figure 5 represents the degree of average load imbalance by communication latency. About 7,000 samples are used at the sampling rate of $50,000 \mathrm{msec}$ $100,000 \mathrm{msec}$. Communication delay is multiples of $10 \mathrm{~m} \mathrm{sec}$. From the figure, load imbalance ranges from 3 to 35 as the latency value changes, but is very high around 81 when no load balancing is applied. In addition, in case of communication time being small, VISITOR ( $¥-$ ) algorithm performs better than VISITOR algorithm. The performance of VISITOR algorithm is improved as the communication latency increases. 


\section{4-3 Neutral Zone}

Neutral zone is a handler for controlling load state of random node and regulating frequency of process migration. Therefore, suitable adjustment of Neutral zone is a key element for influencing the performance of load balancing algorithm. Neutral zone as shown in figure 6 has two neutral widths approximately and these widths represent optimal load state. If this zone gets narrower, the load difference between two nodes decreases picking up many load migration, and thus increases communication costs of load balancing. As the zone width gets larger, the frequency of migration becomes smaller and thus may allocate more time on process execution.

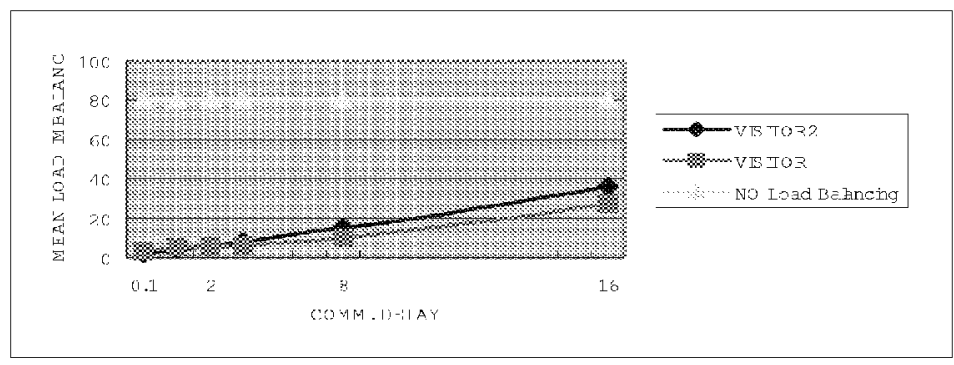

Fig. 5. The change of load imbalance due to communication latency

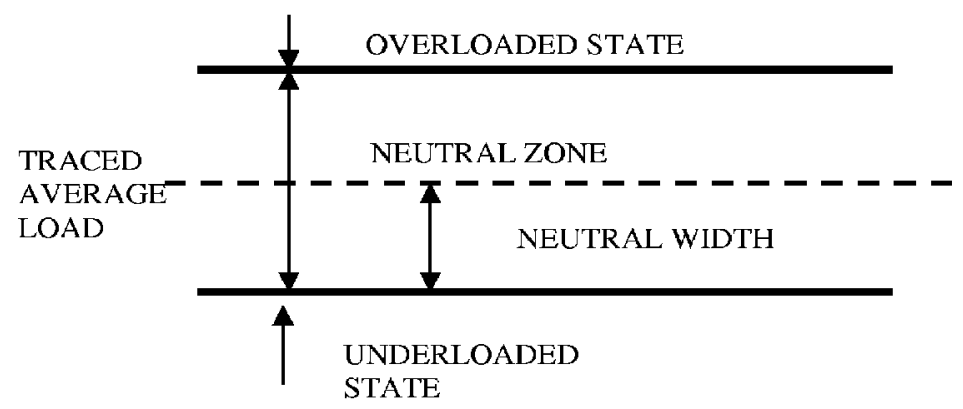

Fig. 6. The relation of neutral zone and load status

\subsection{The Average Response Time}

It is important to measure response time of random process for analyzing the system performance. The simulation uses equation (3) for measurement of average response time that represents the total length of visit from which the process arrives in waiting queue and until it leaves, including remote processing 


$$
\operatorname{MR}(\mathrm{t})=\phi+R(\mathrm{t}) / \mathrm{p}
$$

$R(t)$ represents the response time of random process and $p$ is the number of process. Defined in equation (4) is the average response time of total system.

$$
\operatorname{ASRT}(\mathrm{t})=\xi+[\mathrm{c}+\mathrm{R}(\mathrm{t}) / \mathrm{p}] / \mathrm{m}
$$

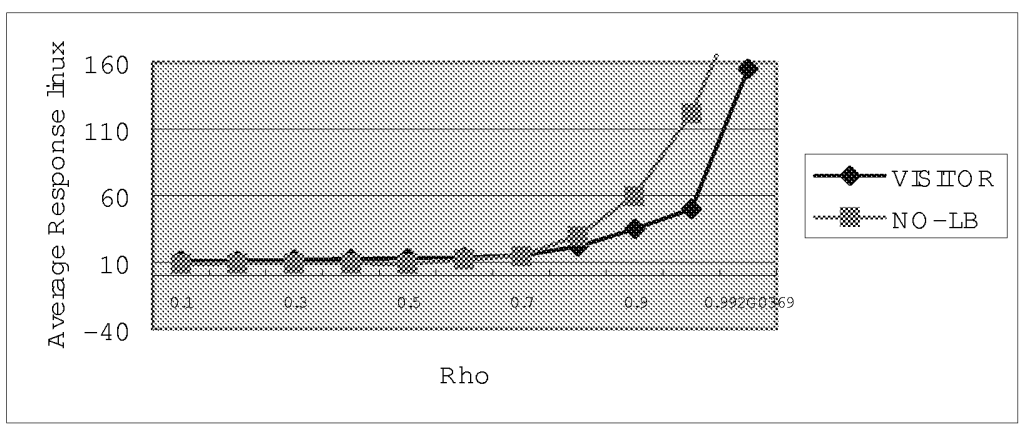

Fig. 7. The average arrival rate versus average service time

Figure 7 indicates that the result of average response time is better than the one when no load balancing algorithm is applied. Especially, when ¥æ is $0.7 \sim 0.8$, the average response time increases sharply while VISITOR incurs a rather smooth increases. Before ¥æapproaches to 0.7, the proposed algorithm shows the worse performance due to various overheads, such as status information exchange, communication latency, message loss, etc.. After ¥æ passes 0.7 , however, VISIOTOR (II) shows stable increases in average response time.

\section{Conclusion and Future Work}

In this paper, different methods of load balancing are presented to remove load imbalance, which occurs in typical distributed system. For this, two algorithms are introduced and analyzed with their performance comparison. With VISIOR (II) algorithm, the global information is achieved with less communication costs, compared with the one in broadcasting mechanism, and the accuracy of the information is proved. The performance, in terms of average response time, is improved about $50 \sim 80 \%$ when $¥ æ$ is $0.9 \sim 0.99$ than when no load balancing algorithm is applied. The communication cost for updating the status information is reduced remarkably because the global 
information is managed with simple message circulation and tracking functions.

"Victim Problem" [1] in VISITOR algorithm, however, can cause poor system performance as decision making at each nodes is combined. For future studies, cooperation and coordination mechanism to reduce the frequency of decision-making will be examined. Also, the recovery and rehabilitation mechanism for reducing the possibility of message loss in communication line will also be studied.

\section{References}

1. Alon, M., Barak, A., Manber, U., "On Dissemination Reliably without Broadcasting," IEEE The $7^{\text {th }}$ International Conf. On Distributed/Computing Systems, pp. 74-81, Sep. 1987.

2. Casavant,T. and Singhal, M., Reading in Distributed Computing Systems, IEEE Computer Society Press, 1994.

3. Chao, L. L., Statistics Methods and Analysis, McGraw-Hill, $2^{\text {nd }}$ Edition, pp. 188-192, 1974.

4. Eager, D. L., et al., "Adaptive Load Sharing in Homogeneous Distributed Systems," IEEE Trans. On Software, Vol. SE-12, No. 5, pp.662-675, May 1986.

5. Kness, C., Matkowsky B. J., Schuss, Z., Tier, C., "Two Parallel Queue with Dynamic Routing," IEEE Trans. on Comm., Vol. COM-34, No. 12, pp. 11701175, Dec. 1986.

6. Krueger, P., Livny, M., "A comparison of Preemptive and Non-Preemptive Load Distributing," The $8^{\text {th }}$ International Conf. On DCS, pp. 123-130, 1988

7. Lin ,F.C.H., Keller, R. M., "The Gradient Model Load Balancing Method," IEEE Trans. on SE, Vol. SE-13, No. 1, Jan. 1987.

8. Ni , L. M., et al., "A Distributed Drafting Algorithm for Load Balancing," IEEE Trans. on Software Engineering, Vol. SE-11, No. 10, Oct. 1985.

9. Smith, R. G., "The Contract Net Protocol: High Level Communication and Control in a Distributed Problem Solver," IEEE Trans. on COMPUTERS, Vol. C-29, No. 12, Dec. 1980.

10. Stankovic, J., “A Perspective on Distributed Computer Systems," IEEE Trans. on Comp. Vol. C-33, No. 12, pp. 1102-1115, Dec. 1984.

11. Stankovic, J., "An Application of Bayesian Decision Theory to Decentralized Control of Job Scheduling," IEEE Trans. on Comp., Vol. C-34, No. 2, pp. 117 130, Feb. 1985.

12. Tay, Y. C. and Pang, HweeHwa, "Load Sharing in Distributed Multimedia-onDemand Systems", IEEE Transactions on Knowledge and Data Engineering, Bol. 12, No. 3, May/June 2000.

13. Tonogai, D., "AI in Operating Systems: An Expert Scheduler," PROGRESS REPROT No. 88,12, Dec. 1988.

14. Zhou, S., Ferrari, D., "A Measurement Study of Load Balancing Performance," IEEE The $7^{\text {th }}$ Conf. on DCS, pp. 490-497, Sep. 1987.

15. Zhou, S., Ferrai, D., "An Experimental Study of Load Balancing Performance," REPORT No. UCB/CSD/87/336, Berkeley, California, Jan. 1987. 